\title{
Complexation of Lysozyme with Adsorbed PtBS-b-SCPI Block Polyelectrolyte Micelles on Silver Surface
}

Aristeidis Papagiannopoulos ${ }^{1 *}$, Anastasia Christoulaki ${ }^{2}$, Nikolaos Spiliopoulos ${ }^{2}$, Alexandros Vradis $^{2}$, Chris Toprakcioglu ${ }^{2}$, Stergios Pispas ${ }^{1 *}$

${ }^{1}$ Theoretical and Physical Chemistry Institute, National Hellenic Research Foundation, 48 Vassileos Constantinou Avenue, 11635 Athens, Greece

${ }^{2}$ Department of Physics, University of Patras, GR 26 500, Greece

*apapagiannopoulos@eie.gr*pispas@eie.gr

\begin{abstract}
We present a study of the interaction of the positively charged model protein lysozyme with the negatively charged amphiphilic diblock polyelectrolyte micelles of poly(tert-butylstyrene)-bsodium (sulfamate/carboxylate)isoprene) (PtBS-b-SCPI) on the surface of silver. The adsorption kinetics are monitored by surface plasmon resonance and the surface morphology by atomic force microscopy. The micellar adsorption kinetics is dictated by two processes and the micellar layer morphology shows that the micelles do not lose their integrity upon adsorption. The complexation of lysozyme with the adsorbed micellar layers depends on the micelles arrangement and density in the underlying layer and lysozyme follows the local morphology of the underlying roughness. When the micellar adsorbed amount is small, the layers show low capacity in protein binding and low resistance in loading. When the micellar adsorbed amount is high the situation is inversed. The adsorbed layers both with or without added protein are found to be irreversibly adsorbed on the Ag surface.
\end{abstract}

\section{INTRODUCTION}

Adsorption of synthetic and biological macromolecules on surfaces and interfaces is a field of wide interest due to its variety of applications[1] and its great impact on the physichochemical properties[2] of the newly created interface i.e. biocompatibilty, stimuli-responiveness and 
stability[3]. Physical adsorption of polyelectrolytes on flat surfaces can be achieved by a variety of driving forces. These can act individually or in combination and may result from electrostatic interactions between the adsorbed macromolecules and the surface and/or hydrophobic effects[4]. Driving forces of this kind may also induce self-assembly in solution with extremely attractive structures, as for example micelles of diblock polyelectrolytes where the hydrophobic parts of the diblock chains aggregate to form a dense hydrophobic core whereas the hydrophilic polyelectrolyte blocks extend in solution to form a highly hydrated corona[5].

Polyelectrolyte micelles containing both hydrophobic and charged groups may interact with surface groups and form physical bonds. An adsorbed polyelectrolyte micelle may keep its solution structure and integrity upon adsorption so that a rough layer forms[6], when the hydrophobic blocks in its core are in a glassy state and they are kinetically frozen. So even if it is energetically favourable for them to release their contacts and create new ones with the surface they remain inside the micelle's core. In the case of a core in a liquid state, the micellar structure may dissolve, so that the cores spread out on the surface and the hydrophilic blocks extend away from the surface to form a flat layer [7].

Proteins interact with polyelectrolytes, both in solution and on surfaces[8] mainly due to electrostatic interactions. This kind of interactions open a field for research towards applications in engineering and medicine, including encapsulation and delivery of drugs, proteins and DNA $[9,10]$. Proteins normally complex with oppositely charged macromolecular chains belonging to spherical micelles [11] in solution, which affects critically the chain conformation even causing intermicellar aggregations[12]. Similarly, on a planar surface coated with a polyelectrolyte layer, incorporation of protein globules is expected to cause conformational changes upon the adsorbed chains[13].

Surface Plasmon Resonance (SPR) is a high sensitivity tool for surface studies and it has been widely utilized for biosensing applications[14]. SPR has also been successfully used for adsorption studies of polyelectrolytes[15] and proteins[16]. In this article we present the adsorption kinetics of poly(tert-butylstyrene)-b-sodium (sulfamate/carboxylate)isoprene) (PtBSb-SCPI) micelles on the silver/water interface and the interactions of the formed layers with the oppositely charged lysozyme. The morphology of the adsorbed layers is investigated by Atomic 
Force Microscopy (AFM). We are primarily interested in understanding the physicochemical and self organization processes taking place in such synthetic/biological hybrid nanosystems.

\section{MATERIALS AND METHODS}

\section{Materials}

The diblock polyelectrolyte poly-(tert-butylstyrene-b-sodium (sulfamate/carboxylate) isoprene), PtBS-b-SCPI, was synthesized by anionic polymerization high vacuum techniques and block selective post-polymerization functionalization reactions [17, 18]. The resulting $M_{\mathrm{w}}$ is $164 \mathrm{~kg} / \mathrm{mol}$ (I=1.03) and the respective weight contents are $12 \%$ for PtBS and $88 \%$ for SCPI. PtBS is a hydrophobic polymer with high glass transition temperature and SCPI is a highly charged polyelectrolyte that contains both strong $\left(\mathrm{SO}_{3}^{-}\right)$and weak $\left(\mathrm{COO}^{-}\right)$charged groups (figure 1). In aqueous solution, PtBS-b-SCPI forms micelles with a core of hydrophobic PtBS blocks and a corona of hydrophilic negativelly charged SCPI blocks. A diblock polyelectrolyte solution (1 $\mathrm{mg} / \mathrm{ml}$ ) was prepared in a $\mathrm{pH} 70.01 \mathrm{M}(\mathrm{NaCl})$ buffer (the same buffer was used to prepare lysozyme solutions) by heating at $60{ }^{\circ} \mathrm{C}$ overnight. This stock solution was used to prepare solutions of the preferred lower concentrations by diluting with the same buffer. The hydrophobic PtBS blocks form a solid "frozen" core (because of the strong hydrophobic nature of PtBS and its high Tg), which is surrounded by the water-soluble hydrophilic polyelectrolyte SCPI blocks. The PtBS-b-SCPI diblock copolymer in aqueous solutions has been studied thoroughly [12] in the past and well-defined core-shell micelles were found to form in aqueous solutions of similar conditions. The hydrodynamic radius of the micelles was $R_{h}=100 \mathrm{~nm}$ and the molecular weigh $M=1.6 \cdot 10^{6} \mathrm{~g} / \mathrm{mol}$, which corresponds to about 10 diblock copolymer chains per micelle.

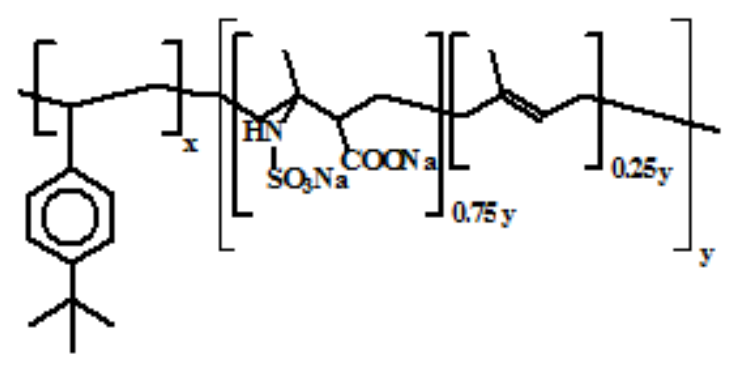


Figure 1: Chemical structure of PtBS-b-SCPI copolymer. The SCPI block contains 75\% monomers with chargeable groups in a random sequence.

Lysozyme (HEWL) with molecular weight $14.7 \cdot 10^{3} \mathrm{~g} / \mathrm{mol}$ was purchased from Fluka and used without further purification. HEWL was dissolved at $0.1 \mathrm{mg} / \mathrm{ml}$ in the $\mathrm{pH} 7 \mathrm{I}=0.01 \mathrm{M}$ buffer and left overnight to equilibrate. The target concentrations were produced by the parent solution by dilution in the same buffer. Strong electrostatic attractions between the polyelectrolyte micelles and the proteins are expected to occur on the surface as it has been proved to happen in solution[12].

\section{Surface Plasmon Resonance Experiments}

The Kretschmann[19] configuration (figure 2) was realized by silver (Ag) films formed on the optically flat face of SF10 equilateral prisms $\left(\mathrm{n}_{\mathrm{SF} 10}=1.723\right)$. The glass films were cleaned by exposure to fresh mixed nitric and hydrocloric acid (1:3 by volume) and then rinsed by water and ethanol. The silver layers $(48-52 \mathrm{~nm})$ were deposited on the glass surface by thermal evaporation of $99.999 \%$ pure silver wire at a base pressure of $1 \cdot 10^{-6}$ Torr and at a deposition rate of 0.15 $\mathrm{nm} / \mathrm{s}$. The prism remains in vacuum for at least half an hour before being removed from the evaporation chamber. The light source used for SPR was a He-Ne laser beam $(\lambda=632.8 \mathrm{~nm})$. A polarizer is used for the p-polarization of the beam since surface plasmons are excited only by the p-component of electromagnetic waves, parallel to the metal surface[20]. The solutions are loaded in a PTFE cell which is sealed on the metal-deposited surface of the film. The temperature of the solution is monitored by a Teflon-coated thermocouple immersed into the solution. The experiments were performed at room temperature. 


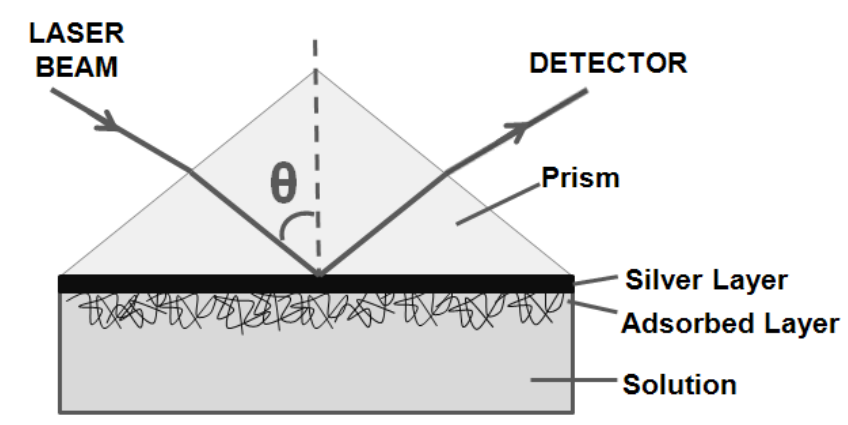

Figure 2: Representation of the SPR experiment geometry used in this study.

\section{Surface Plasmon Resonance Data Analysis}

Electromagnetic surface waves along a metal/dielectric interface can be formed under certain conditions which are called surface plasmons [21]. The dispersion relation in surface plasmons is expressed by equation 1 .

$\omega\left(k_{x}\right)=\frac{\omega}{c} \cdot\left(\frac{\varepsilon_{1} \cdot \varepsilon_{2}}{\varepsilon_{1}+\varepsilon_{2}}\right)^{1 / 2}$

Where $\omega$ is the frequency of the surface plasmons, $k_{x}$ is the x-component of the incident wave vactor $\varepsilon_{1}$ the complex dielectric constant of the metal, $\varepsilon_{2}$ the dielectric constant of the surrounding medium and $c$ the speed of light in vacuum[22].

The dispersion relation of a surface plasmon formed on a thin metal film is influenced by both the characteristics of the film (thickness and dielectric constant) and the characteristics of any substances in the vicinity of the film. This way, any adsorbed layer causes alterations on the reflectivity versus angle of incidence curve compared to the curve from the film with no adsorbed layer [20]. In more detail, a system of $N$ layers with different dielectric constants $\left(\varepsilon_{1}, \varepsilon_{2}, \ldots, \varepsilon_{N}\right)$ can be described[23] by an equal number of characteristic matrices (equation 2 ). 


$$
M(n)=\left(\begin{array}{cc}
\cos \left(k_{0} d_{n} \sqrt{\varepsilon_{n} \mu_{n}} \cos \theta_{n}\right) & -\frac{i}{p_{n}} \sin \left(k_{0} d_{n} \sqrt{\varepsilon_{n} \mu_{n}} \cos \theta_{n}\right) \\
-i p_{n} \sin \left(k_{0} d_{n} \sqrt{\varepsilon_{n} \mu_{n}} \cos \theta_{n}\right) & \cos \left(k_{0} d_{n} \sqrt{\varepsilon_{n} \mu_{n}} \cos \theta_{n}\right)
\end{array}\right) \text { for } n=1, \ldots, N(2)
$$

Where $p_{n}=\sqrt{\frac{\varepsilon_{n}}{\mu_{n}}}$ and $\mu_{n}$ is the magnetic permittivity of layer $n$ which is taken equal to unity for all the materials used in this study. $d_{n}$ is the thickness of layer $n$. $k_{0}$ is the wave number corresponding to the wavelength $\left(\lambda_{0}\right)$ of the incident light $\left(k_{0}=\frac{2 \pi}{\lambda_{0}}\right)$. The angle of incidence $\left(\theta_{n}\right)$ is given by $\cos \theta_{n}=\sqrt{1-\frac{\varepsilon_{1}}{\varepsilon_{f}} \sin ^{2} \theta_{n}}$, where $\theta$ is the angle of insidence on the silver surface (figure 2) and $\varepsilon_{f}$ is the dielectric constant of the semi-infinite medium in contact with the $N^{t h}$ layer. In our case $\varepsilon_{f}$ is the dielectric constant of the buffer solution.

Eventually a $2 \times 2$ matrix that represents the whole stack of layers is calculated by equation 3 .

$M_{\text {system }}=\prod_{n=1}^{N} M(n)$

The reflectivity $(R)$ as a function of the angle of incidence $(\theta)$ is given by the matrix elements of $M_{\text {system }}$ (equation 4).

$R=\left|\frac{\left(M_{\text {system }}^{11}+M_{\text {system }}^{12} p_{N}\right) \cdot p_{1}-\left(M_{\text {system }}^{21}+M_{\text {system }}^{22} p_{N}\right)}{\left(M_{\text {system }}^{11}+M_{\text {system }}^{12} p_{N}\right) \cdot p_{1}+\left(M_{\text {system }}^{21}+M_{\text {system }}^{22} p_{N}\right)}\right|$

A typical SPR reflectivity curve (inset of figure 3) shows a characteristic minimum where the $k_{x}$ component of the incident wave vector matches the surface plasmon wave vector. Additionally there is a characteristic feature at lower angle signifying the critical angle $\left(\theta_{\text {crit }}\right)$ for total internal reflection. 
In this article we study adsorption on the $\mathrm{Ag} / \mathrm{H}_{2} \mathrm{O}$ interface. Before adding a solution to the cell, a reference measurement was taken for buffer in contact with Ag. Experiments of this kind were performed for longer than 12 hours in order for the stability of the Ag films to be guaranteed. Adsorption on silver has been performed for SPR in several studies in the past[24, 25]. The Ag layer in contact with buffer was modeled by a 1-layer system where the thickness and dielectric constant of the Ag layer are the fitting parameters. The fitted parameters of this solute-free system are held fixed in the fitting procedures of the glass/Ag/adsorbed layer/solution structure, assuming that the Ag films do not experience any alteration during the adsorption procedures. In this case a 2-layer model is used. In figure 3 the fitting quality of the glass/Ag/buffer and glass/Ag/adsorbed layer/solution after $4 \mathrm{~h}$ and $8 \mathrm{~h}$ from adding the micellar solution is shown. Typical values for complex dielectric constant and thickness of the silver layer were $\varepsilon_{A g}=$ $-16.2+0.58 i$ and $d_{A g}=49 \mathrm{~nm}$ which are in agreement with literature values[19, 26] and the expected deposited thicknesses. The details about the adsorbed layer will be analyzed in the discussion section.

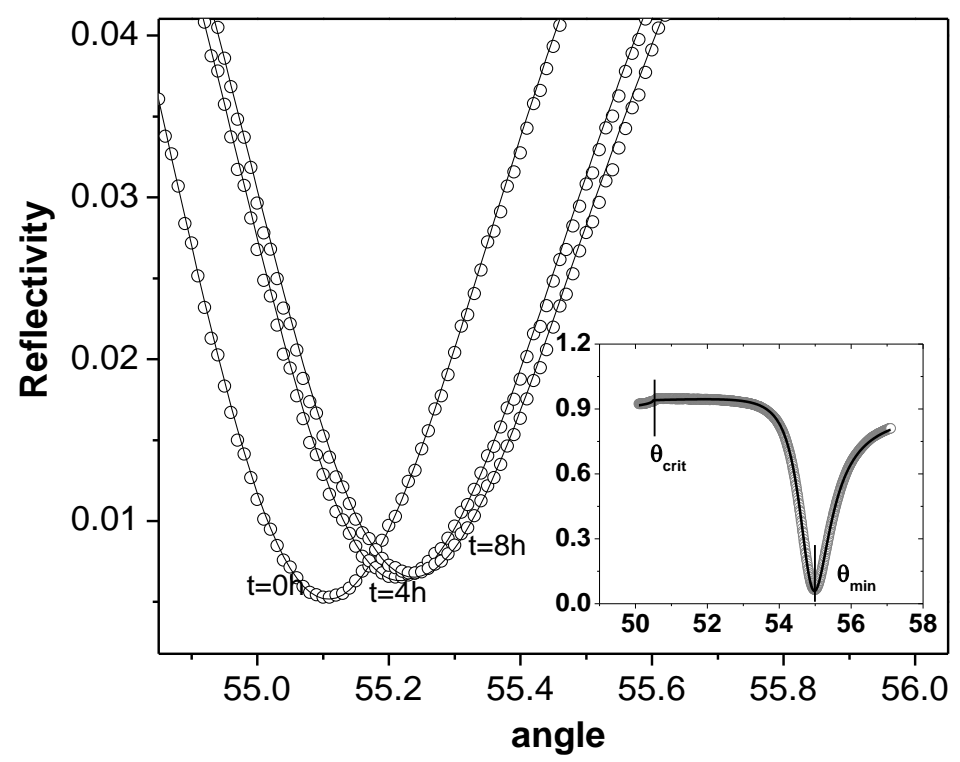

Figure 3: Characteristic SPR curves (near the SPR minimum) of the Ag/water interface for 0, 4 and $8 \mathrm{~h}$ of PtBS-b-SCPI micelles adsorption. The continuous lines are fitting curves. Inset: fullscale experimental SPR curve from the $\mathrm{Ag} /$ water interface (continuous line is the fitting curve). 


\section{Atomic Force Microscopy}

Scanning Probe Microscope was used in the tapping mode for imaging the surface morphology. Ag layers were deposited on glass slides that had been treated by exactly the same deposition protocol as used for SPR on prisms. A twin adsorption protocol as the one used for SPR (see discussion section) was followed for adsorption on Ag layers deposited on glass slides. The deposition of $\mathrm{Ag}$ slides was performed under exactly the same conditions as the deposition on prisms. After the end of every adsorption protocol the surface was gently rinsed with buffer solution in order to remove any non-adsorbed residues and the surfaces were shaken in distilled water to remove any left buffer solution. This is a quasi-dried state since the hydrophilic adsorbed layer may still be hydrated during measurement.

AFM provides information on the structure of adsorbed layers and thin films[27] on the $x-y$ plane. This is quantitatively described by the height of the surface profile [28] as a function of position on the plane $z(x, y)$. A measure of the degree of roughness of a surface is the rms roughness $(\sigma)$ given by equation (5).

$\sigma=\left(\frac{1}{l} \int_{l}(z(l)-\bar{z})^{2} d l\right)^{1 / 2}$

Equation 5 is an average over a trajectory (l) when a section analysis is performed (see discussion about AFM results) or an average over the 2-D surface when 2-D analysis is performed. Deeper physical insight into the roughness morphology can be obtained by looking at the power spectral density $P_{2 D}\left(s_{x}, s_{y}\right)=\frac{1}{A}\left|\int_{A}(z(x, y)-\bar{z}) e^{2 \pi i\left(s_{x} x+s_{y} y\right)} d x d y\right|^{2}$ where the integral is over an area $A$ and $\bar{Z}$ is the average height of the surface. Integrating in polar coordinates $\left(s=\left(s_{x}^{2}+s_{y}^{2}\right)^{1 / 2}\right.$ and $\left.\tan \varphi=\frac{s_{y}}{s_{x}}\right)$ on the inverse plane[28] the radial power spectral density (referred to as the PSD from now on) is obtained: 
$P(s)=\frac{1}{2 \pi} \int_{0}^{2 \pi} P_{2 D}(s, \varphi) d \varphi$

The PSD function provides information about the characteristic length scales and the self-similar structure of surfaces.

\section{RESULTS AND DISCUSSION}

Surface plasmon resonance (SPR) uses the emitted plasmons formed on the interface of a thin metallic film and an insulating material (normally a glass) when light is totally reflected on the glass/metal interface. As the angle of the incident light increases, the reflected intensity passes through a minimun which is the signature of the resonance[29]. The sharpness of the minimum depends on the film thickness and the quality of the interfaces. Adsorption of substances on the metal surface from solution are being detected by shifts of the minimum reflectance angle.

The results from a PtBS-b-SCPI solution at $0.5 \mathrm{mg} / \mathrm{ml}$ in contact with $\mathrm{Ag}$ are shown in figure 4. It is a clear sign of a forming adsorbed layer that there is a systematic shift of the minimum to higher angles and higher reflectivity values as a function of time. Adsorption of our micelles on Ag from water possibly happens because of the presence of hydrophobic units within the polyelectrolyte chains forming the micellar corona (figure 1) which tend to decrease their contacts with water. The hydrophobic micellar core would also preferably adsorb on the $\mathrm{Ag}$ surface although this would need a strong conformational change of the surrounding corona and maybe desorption of seceral segments of the polyelectrolyte chains. Since adsorption of polyelectrolytes with hydrophobic blocks is normally irreversible we would expect the hydrophobic core of the micelles to be either suspended away from the surface or collapsed on top of the adsorbed polyelectrolyte chains.

The increase in the minimum reflectance cannot be modeled by the assumption of a layer of real dielectric constant because this can only shift the position of the minimum along the horizontal axis. There are several reasons that the minimum can shift to higher reflectivity values. Theoretically the formation of an adsorbed layer with real dielectric constant shifts the minimum 
only along the horizontal axis. The presence of an imaginary component is needed for the vertical shift of the minimum to higher reflectivity values. This could be then attributed to conductive components embedded into the adsorbed layer. A good example is polyelectrolyte multilayers that contain Au nanoparticles [30] where is clear evidence of this kind of minimum's shift and another one is the systematic investigation of films of absorbing dielectrics where conducting polymers are used [31]. In our case there is no such conducting component and any ionic conductivity effects due to the counterions are expected to be negligible [32]. The value of the minimum reflectance is also affected by the roughness of the adsorbed layers[33] which causes scattering of the visible light. The off-specular reflections caused by surface irregularities [34] with hights comparable to the incident light's wavelength give significant scattered light that cannot be separated from the specularly reflected light. Since the shift in the reflectance minimum happens during adsorption we conclude that it is due to off-specular scattering from the forming micellar layer that produces inhomogeneities on the $x-y$ plane. We model this shift by a constant background factor added to the calculated reflectivity. At any case, the value of this background correction is lower than $4 \cdot 10^{-3}$. Modeling this shift by an effective imaginary part of the adsorbed layer's dielectric constant instead of a constant background the results of the adsorbed layer's fitting parameters are not significantly changed. This scattering maybe caused by micellar scattering, in case the micelles do not lose their integrity upon adsorption and give rise to height fluctuations due to roughness. As it will be shown in the AFM results the produced surfaces are indeed rough.

The situation is qualitatively similar to the one of the $0.5 \mathrm{mg} / \mathrm{ml}$ solution, for the PtBS-b-SCPI solutions at $0.1 \mathrm{mg} / \mathrm{ml}$ and $0.3 \mathrm{mg} / \mathrm{ml}$ in contact with $\mathrm{Ag}$ i.e. systematic evolution of the SPR minimum to higher angles and values (SPR data not shown). 


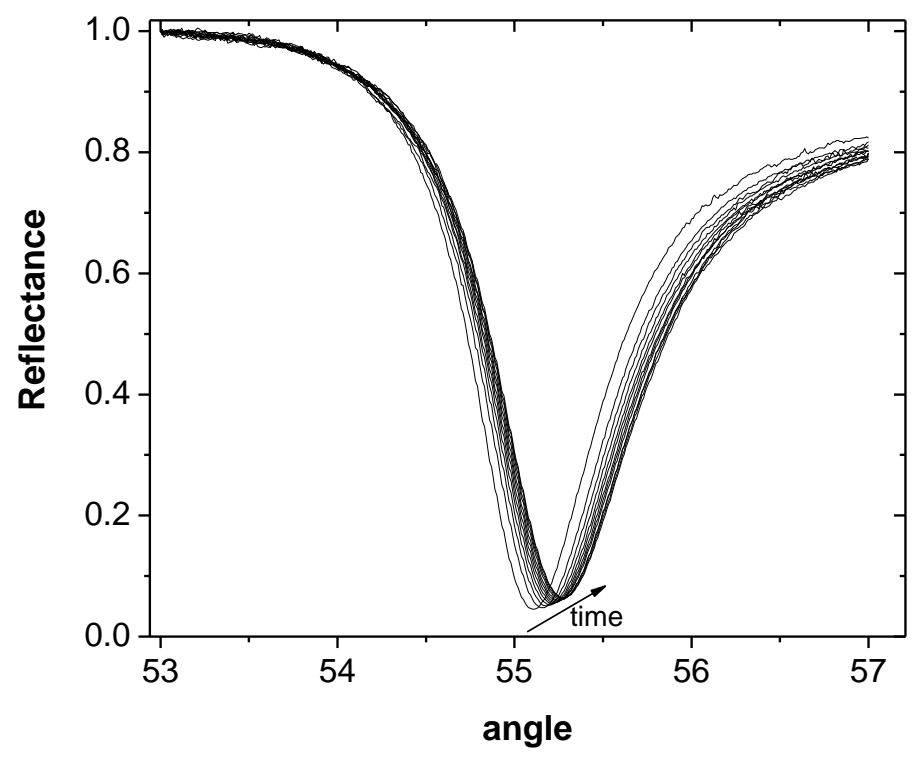

Figure 4: Experimental SPR curves from PTBS-b-SCPI micelle solutions in contact with Ag layer. Time starts when the solution is loaded to the cell and continues for about $800 \mathrm{~min}$. One curve every $60 \mathrm{~min}$ is presented.

The dielectric constant of the adsorbed layer is calculated by:

$$
\varepsilon_{\text {layer }}=\varphi_{p o l} \cdot n_{\text {pol }}^{2}+\left(1-\varphi_{p o l}\right) \cdot n_{w a t e r}^{2}
$$

The refractive indices of PtBS-b-SCPI (calculated by the individual components) and water are $n_{\text {pol }}=1.5$ and $n_{\text {water }}=1.332$ respectively. The absolute refractive index of water is obviously altered by the presence of polymer (or protein in the following discussion) and salt ions. However, due to the low concentrations investigated, there is no detectable difference expected.

In the fitting procedures a normalization factor $(N)$, the thickness $(d)$, the volume fraction $\left(\varphi_{p o l}\right)$ of the adsorbed layer and the constant background are the optimization parameters. The 
thickness and volume fraction of the polymer in the adsorbed layer were found to be mutually dependent as in other studies [20]. This is because the increase in both thickness or polymer volume fraction causes the minimum angle to shift to higher values without a detectable change in the curve shape for the observed layers. For this reason the output parameter of the fit that describes the adsorbed layer is solely the adsorbed amount $\Gamma$ given by:

$$
\Gamma=\rho_{p o l} \cdot \varphi_{p o l} \cdot d
$$

The adsorbed amount of PtBS-b-SCPI on the Ag/water interface is shown in figure 4 for $0.1,0.3$ and $0.5 \mathrm{mg} / \mathrm{ml}$. Adsorption increases at a high rate up to about 200min and at a decreased rate at higher times. The time-scale of the adsorption is in the order of 10 hours which is common for adsorption of polyelectrolyte systems [1, 35, 36]. This kind of kinetics i.e. a fast initial stage followed by a slow stage has been observed also for polyampholyte micelles on silicon[6].

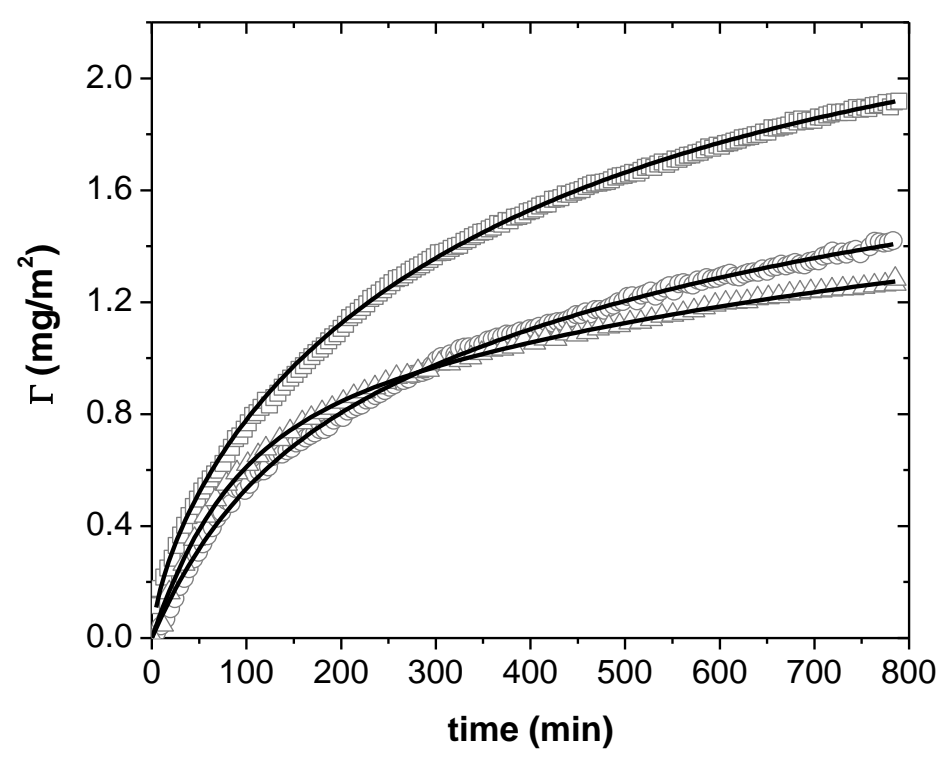


Figure 5: Adsorbed amount as a function of time for adsorption on the $\mathrm{Ag} / \mathrm{H}_{2} \mathrm{O}$ interface for PtBS-b-SCPI 0.5 (squares), 0.3 (circles) and 0.1 (triangles) mg/ml solutions. The continuous lines are fits with equation 9.

The adsorbed amount as a function of time i.e. the adsorption kinetics of the PtBS-b-SCPI micelles on silver was modeled by a linear combination of two first-order Lagergren models (equation 9). This model represents adsorption that is dominated by two processes. One fast initial adsorption process (short characteristic time) and one later adsorption process (longer characteristic time).

$\Gamma(t)=\Gamma_{1} \cdot\left(1-\exp \left(-\frac{t}{\tau_{1}}\right)\right)+\Gamma_{2} \cdot\left(1-\exp \left(-\frac{t}{\tau_{2}}\right)\right)$

The fitting parameters of equation 8 are the asymptotically reached adsorbed amounts of the two processes $\left(\Gamma_{1}\right.$ and $\left.\Gamma_{2}\right)$ and the corresponding fast and slow adsorption characteristic times ( $\tau_{1}$ and $\tau_{2}$ ). This kind of model has also been used for adsorption of polyelectrolytes in the form of a modified Johnson-Mehl-Avrami equation $[37,38] \quad \Gamma(t)=\Gamma_{1} \cdot\left(1-\exp \left(-\frac{t}{\tau_{1}}\right)\right)+\Gamma_{2}$. $\left(1-\exp \left(-\left(\frac{t}{\tau_{2}}\right)^{a}\right)\right)$ where the exponent $a$ is an additional fitting parameter. In our case $a=1$.

For sake of the following discussion we label the three different adsorbed layers on account of their total adsorbed amounts (table 1) as "low", "intermediate" and "high". The adsorbed amount at infinite time $\left(\Gamma_{t o t}=\Gamma_{1}+\Gamma_{2}\right)$ increases as a function of solution concentration which is normal behavior for unsaturated surfaces[35]. The characteristic fast time-scale $\left(\tau_{1}\right)$ is nearly the same for the low and intermediate coverage, although for the low surface coverage the short-time kinetics is evidently faster compared to the intermediate overage (up to $250 \mathrm{~min}$, figure 5). In any case, it is clear that for the high concentration solution the initial stage of adsorption has a faster characteristic time. This is in agreement with the Langmuir model for adsorption kinetics 
where the rate constant increases with the solution concentration[39]. When the coverage of the surface is relatively low, the adsorption process is dominated by the bulk solution concentration and it becomes faster as the concentration increases.

The slow adsorption process (described by $\tau_{2}$ ) becomes slower as a function of surface coverage. This points to the hypothesis that this process is driven by in-plane micellar rearrangements[40] that are necessary for the incoming ones to be accommodated and/or steric and electrostatic repulsions between the incoming and the adsorbed micelles. In a study of charged $\mathrm{ABC}$ triblock terpolymer micelle adsorption, a kinetic barrier upon adsorption was observed caused by hardsphere jamming limit on the surface[41]. The in-plane micellar rearrangements are obviously slower when the surface coverage is higher and the adsorbed micelles are highly overlapping. Furthermore, the incoming micelles experience a higher barrier resistance [42] as the surface coverage increases. A measure that gives insight to the structure of the formed layers is the overlap surface coverage $\Gamma^{*}$. Assuming uniform arrangement of micelles on the surface there are two extreme cases, the one for very low coverage where the micelles are far away from one another so that no interaction within the adsorbed layer is possible, and the other where the micelles are overlapping and their conformation may be strongly affected by the neighboring micelles. The $\Gamma^{*}$ value that defines the cross-over of the two regimes, is the one for which the micelles fully cover the surface but do not overlap and it can be estimated by:

$\Gamma^{*} \approx \frac{M / N_{A}}{\pi R_{h}^{2}}$

Using the documented values for molecular weight $(M)$ and hydrodynamic radius $\left(R_{h}\right)$ of the formed micelles in solution under the conditions of this study[12] the resulting critical coverage is $\Gamma^{*} \approx 0.1 \mathrm{mg} / \mathrm{m}^{2}$. It is clear from the values of $\Gamma_{t o t} / \Gamma^{*}$ (table 1) that in the formed layers the micelles are well overlapping. Additionally, the overlapping coverage is reached quite soon in the adsorption experiments (within the first 10-15 min of adsorption) which explains the absence of a diffusion dominated adsorption process even at short times. The average in-plane distance between two neighboring micelles on the surface may be estimated by $r_{m} \approx\left[\Gamma_{t o t} /\left(M \cdot N_{A}\right)\right]^{-\frac{1}{2}}$. 
For the adsorbed amounts of this study we estimate the average intermicellar distance to be $\mathrm{r}_{m} \approx 40 \mathrm{~nm}$. The core-radius $\left(\mathrm{r}_{\text {core }}\right)$ of the micelles can be calculated under the assumption that the core is solid PtBS, from $m_{\text {core }} \approx \rho_{P t B S} \cdot \frac{4}{3} \pi \mathrm{r}_{\text {core }}^{3}$ where $\rho_{P t B S}$ is the bulk density of PtBS and $m_{\text {core }}$ is the mass of the core, which is known from the micellar mass and the percentage of PtBS in the diblock copolymer. The resulting radius is $\mathrm{r}_{\text {core }} \approx 4 \mathrm{~nm}$ concluding that the adsorbed layers are in the "soft" overlaping and not in the "hard" overlaping state i.e. the overlapping is between the micellar coronas while the cores are well-separated.

Table 1: Optimized parameters for the three adsorbed micellar layers by fitting with the model of equation 9.

\begin{tabular}{|c|c|c|c|c|c|c|c|}
\hline $\begin{array}{c}\text { concentration } \\
(\mathbf{m g} / \mathbf{m l})\end{array}$ & $\boldsymbol{\Gamma}_{\mathbf{1}}\left(\mathbf{m g} / \mathbf{m}^{\mathbf{2}}\right)$ & $\boldsymbol{\tau}_{\mathbf{1}}(\mathbf{m i n})$ & $\left.\boldsymbol{\Gamma}_{\mathbf{2}} \mathbf{~ m g} / \mathbf{m}^{\mathbf{2}}\right)$ & $\boldsymbol{\tau}_{\mathbf{2}}(\mathbf{m i n})$ & $\begin{array}{c}\boldsymbol{\Gamma}_{\text {tot }} \\
\left(\mathbf{m g} / \mathbf{m}^{\mathbf{2}}\right)\end{array}$ & $\boldsymbol{\Gamma}_{\text {tot }} / \boldsymbol{\Gamma}^{*}$ & coverage \\
\hline 0.1 & $0.67 \pm 0.03$ & $75 \pm 4$ & $0.90 \pm 0.04$ & $710 \pm 120$ & 1.57 & 15.7 & low \\
\hline 0.3 & $0.50 \pm 0.04$ & $87 \pm 6$ & $1.23 \pm 0.02$ & $590 \pm 70$ & 1.73 & 17.3 & intermediate \\
\hline 0.5 & $0.32 \pm 0.02$ & $28 \pm 2$ & $1.74 \pm 0.02$ & $340 \pm 6$ & 2.06 & 20.6 & high \\
\hline
\end{tabular}

After the $800 \mathrm{~min}$ run of every adsorption test the solution was removed and the cell was rinsed with fresh buffer. A 240min measurement of the adsorbed layer in contact with buffer was run and no detectable change was observed on the adsorbed layers, which proves the PtBS-b-SCPI micelles adsorbed irreversibly on Ag forming stable layers. Additionally, another 800min adsorption experiment was performed to form another high coverage layer (from $0.5 \mathrm{mg} / \mathrm{ml}$ solution concentration) on $\mathrm{Ag}$ in order for its stability to be tested in physiological ionic strength (i.e. buffer $0.15 \mathrm{M} \mathrm{NaCl}$ ). Similarly to the buffer with low added salt $(0.01 \mathrm{M})$, the layer was found to be stable after addition of $0.15 \mathrm{M} \mathrm{NaCl}$ (tested for longer than 240min).

The interaction of the adsorbed PtBS-b-SCPI adsorbed micelles with lysozyme was tested by a titration-like protocol: after the micelle adsorption test was run (for $\sim 800 \mathrm{~min}$ ) the cell was emptied from the micellar solution and gently rinsed with fresh buffer. Subsequently the titration experiment took place. Interaction with successive solutions of lysozyme at concentrations of 


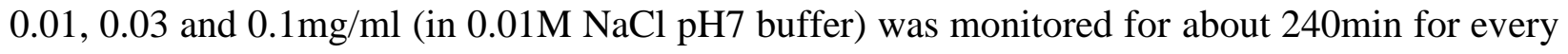
concentration. The cell was gently rinsed with buffer between changes of lysozyme solutions. The SPR curves had qualitatively similar response compared to the adsorption of the polyelectrolyte micelles. The SPR reflectance minimum shifted to higher angles as a function of time which was a signature of the complexation of lysozyme with the interface. The data treatment and fitting were identical to the micelle adsorption case except that now the dielectric constant of the formed layer contains contributions from both micelles and lysozyme:

$$
\varepsilon_{\text {layer }}=\varphi_{\text {pol }} \cdot n_{\text {pol }}^{2}+\varphi_{\text {lyso }} \cdot n_{\text {lyso }}^{2}+\left(1-\varphi_{\text {pol }}-\varphi_{\text {lyso }}\right) \cdot n_{\text {water }}^{2}
$$

For the fits of the mixed layers the adsorbed amount of the micelles is assumed to be unchanged upon interaction with lysozyme and this way the only non-fixed fitting parameters are the volume fraction of lysozyme $\left(\varphi_{\text {lyso }}\right)$ and layer thickness $(d)$, which provide the mass of lysozyme per unit area in the adsorbed layer in a similar manner as in the case of the micellar adsorption (equation 2). The refractive index of lysozyme is $n_{\text {lyso }}=1.516$. We will address to the amount of lysozyme in the layer either as adsorbed or as complexed lysozyme amount. 

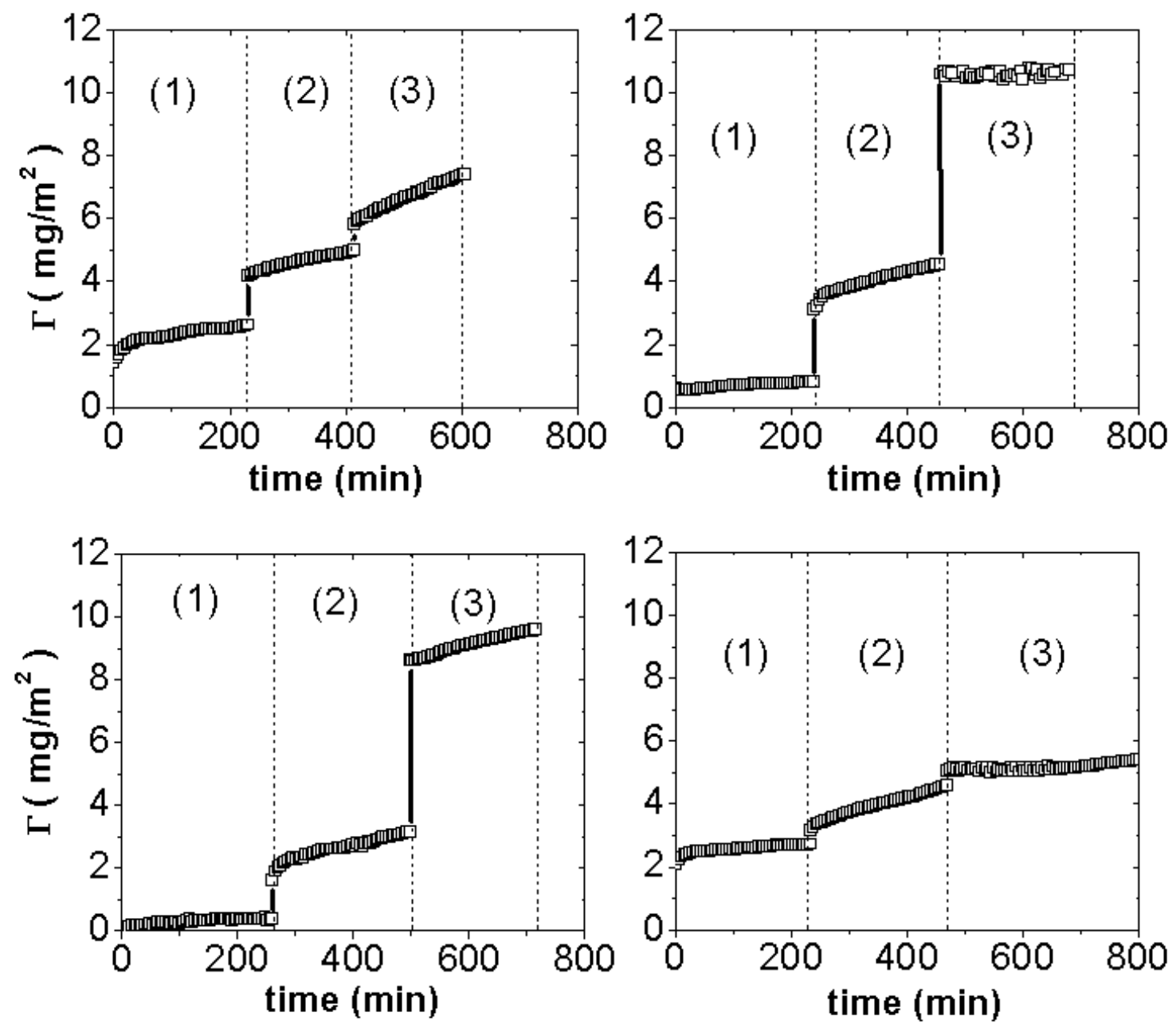

Figure 6: Complexed amount of lysozyme with layers of adsorbed PtBS-b-SCPI micelles as a function of time for layers formed by solution of "low" (top-left), "intermediate" (top-right) and "high" (bottom-left) coverage at pH7 0.01M NaCl and "high" (bottom—right) coverage at pH7 $0.15 \mathrm{M} \mathrm{NaCl}$. The different concentrations of the lysozyme solutions (see protocol discussion) are separated by vertical dashed lines with labels: (1) for $0.01 \mathrm{mg} / \mathrm{ml}$, (2) for $0.03 \mathrm{mg} / \mathrm{ml}$ and (3) for $0.1 \mathrm{mg} / \mathrm{ml}$ lysozyme.

Figure 6 shows the complexation kinetics of lysozyme on the pre-adsorbed PtBS-b-SCPI micelle layers. In most of the cases complexation occurs virtually instantaneously (in the time scale of the observation) as seen by the discontinouities of the complexed amounts when the protein 
concentration changes. There are also signs of on-going (un-saturated complexation) where the complexed amount increases systematically with time for a single protein solution concentration experiment. In any case we believe it is safe to assume that the abrupt increase of the complexed amount of lysozyme between successive runs is due to the increased protein concentration between successive runs.

In the case of $\mathrm{pH} 7$ and $0.01 \mathrm{M} \mathrm{NaCl}$ we observe that the adsorption of protein at step (1) of the runs $(0.01 \mathrm{mg} / \mathrm{ml}$ protein solution in the reservoir) is $2.4 \mathrm{mg} / \mathrm{ml}$ for the low coverage layer, 0.7 $\mathrm{mg} / \mathrm{ml}$ for the intermediate coverage layer and $0.35 \mathrm{mg} / \mathrm{ml}$ for the high coverage layer. During step (2) $(0.03 \mathrm{mg} / \mathrm{ml}$ protein solution in the reservoir) the adsorbed protein amount is $4.9 \mathrm{mg} / \mathrm{ml}$ for the low coverage layer, $4.6 \mathrm{mg} / \mathrm{ml}$ for the intermediate coverage layer and $3.2 \mathrm{mg} / \mathrm{ml}$ for the high coverage layer. Finally, during step (3) (0.1 mg.ml protein solution in the reservoir) the adsorbed protein amount is $7.3 \mathrm{mg} / \mathrm{ml}$ for the low coverage layer, $10.6 \mathrm{mg} / \mathrm{ml}$ for the intermediate coverage layer and $9.7 \mathrm{mg} / \mathrm{ml}$ for the high coverage layer. This means that for low surface coverage, lysozyme adsorbs strongly for low contents in solution whereas for higher coverages lysozyme does not show strong binding at low solution protein contents. As protein solution content increases, higher coverages show higher ability for complexation.

A protein globule experiences a great variety of interactions when it enters the environment of an adsorbed polyelectrolyte layer. The electrostatic interaction with the charged layer is non-trivial due to the patches of positive and negative charges on the protein globule. The exchange of polyelectrolyte counterions with the multivalent protein gives a high entropic gain which is proved to drive the association of proteins even with like-charge polyelectrolytes [8]. The steric/electrostatic interactions between the monomers, force the polyelectrolyte chains to stretch away from the surface especially in the case of end-attached chains (planar macromolecular brushes). When a globule enters a macromolecular brush, steric interactions between chain monomers and protein globules may require the swelling of the adsorbed layer, creating a potential barrier to protein adsorption[43]. Granick et al. [44] studied the adsorption of the negatively charged human serum albumin (HSA) on adsorbed layers of the positively charged poly-4-vinylpyridine (QPVP). The layers were developed either in the statistically adsorbed or in the chain-end grafting (brush) configuration. Equilibrium adsorbed amounts of proteins were higher in the case of the brushes (for similar QPVP surface coverages) and this was attributed to 
the fact that the brush configuration has more charged segments available for binding with proteins compared to the statistically adsorbed chains which have numerous contacts with the surface and also form closed loops. The "stricking" feature was that the kinetics was slower for the brush case and this was attributed to steric effects: a brush produces smaller and deeper pores while statistically-adsorbed macromolecules show shallow and wider pores. A similar effect was observed in the adsorption isotherms where the normalized adsorbed amount versus HSA solution content was more abrupt in the case of the statistically adsorbed layer. The initially complexed proteins produce a barrier to the incoming ones and an osmotic pressure increase is needed for them to move further inside so that new ones are incorporated. [44]

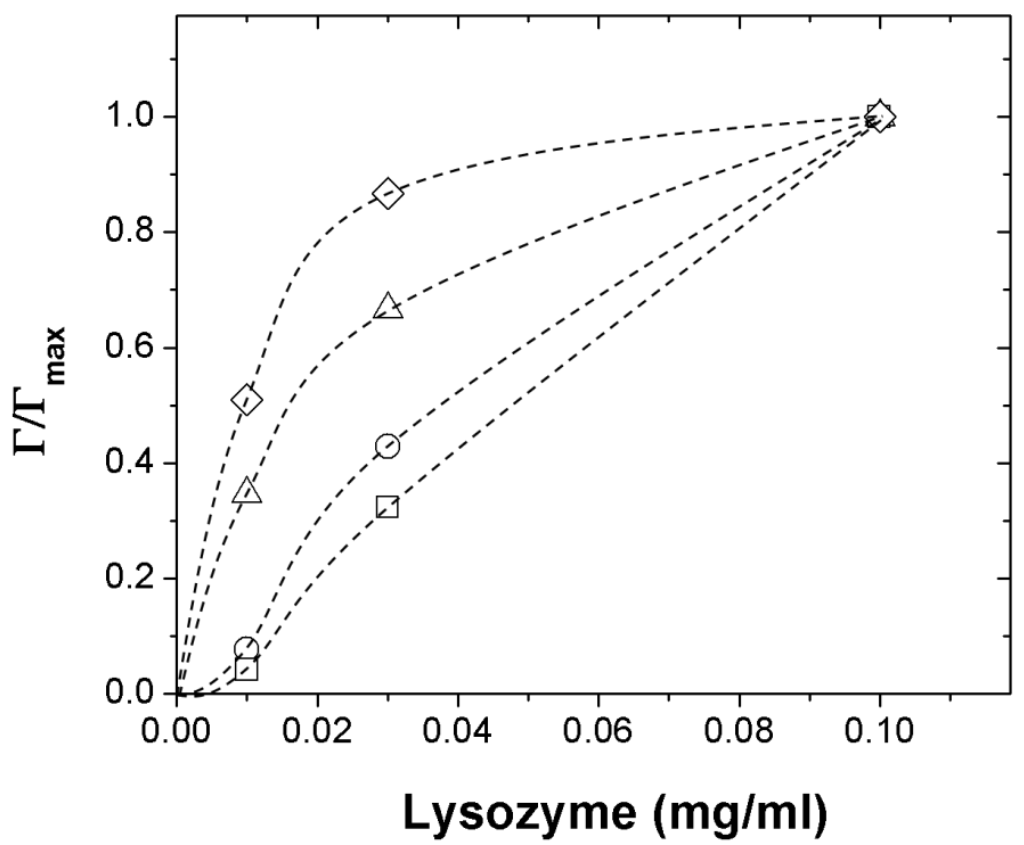

Figure 7: Normalized complexed amount of lysozyme with layers of adsorbed PtBS-b-SCPI micelles as a function of lysozyme solution content for "high" (squares), "intermediate" (circles), "low" (triangles) and "high" with $0.15 \mathrm{M}$ salt (triangles) coverage layers. The dashed lines are guides to the eye. 
When salt is added to the solution a less extended conformation is expected from the adsorbed polyelectrolyte chains[36].

A qualitatively similar behavior is observed in our case (figures 6 and 7). We conclude that in the case of "low" coverage the conformation of the micelle's corona chains is nearer to the statistical adsorption where a lot of contacts exist between the chains and the surface whereas for "high" coverage the situation is closer to the extended brush conformation. This happens because at low coverage the corona chains have enough space to spread on the surface, in contrast to the high coverage where they interact strongly with chains of other micelles and are forced to extend towards the solution. This way the protein binding capacity and the resistance to loading increases as we move from low to high coverage (figure 6 and 7 at $0.01 \mathrm{M}$ salt). When salt content is increased to $0.15 \mathrm{M}$ the behavior of a highly covered layer is on the other extreme of the low coverage layer with low salt content (figure 6 and 7 at $0.15 \mathrm{M}$ salt). This proves that when the electrostatic interactions are weakened the corona chains can create contacts with the surface and decrease their loading capacity and resistance.

The complexed layers (as the micellar ones) proved to be stable upon exchanging the protein solution with buffer solution for more than $8 \mathrm{~h}$ as it was shown by SPR measurements (figure 8). The resonance curve did not show any detectable shift during the $8 \mathrm{~h}$ experiment (note the inset of figure 8). 


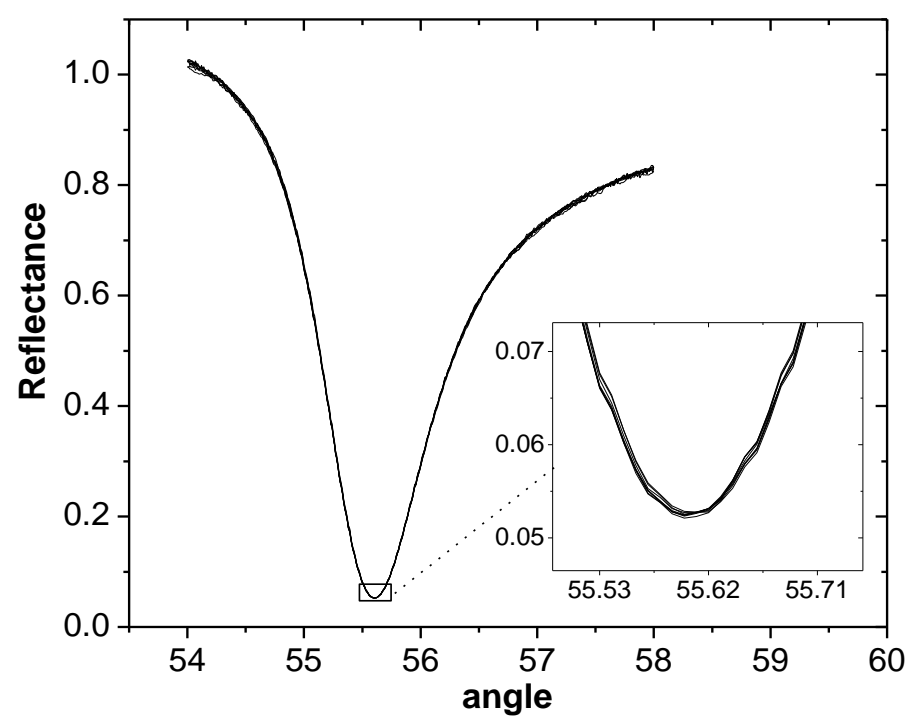

Figure 8: Stability test of the PtBS-b-SCPI high coverage layer complexed with lysozyme in contact with fresh buffer. The 8 curves with $1 \mathrm{~h}$ interval between one another appear to be one single curve. Inset: magnification of the data around the SPR minimum.

A control experiment of adsorption of lysozyme $(0.03 \mathrm{mg} / \mathrm{ml}$ solution concentration) gave an adsorbed amount of $\sim 2.0 \mathrm{mg} / \mathrm{m}^{2}$ in a $240 \mathrm{~min}$ experiment. This shows that the coating with PtBSb-SCPI micelles provides tailor-making of the surface properties in relation to protein binding and immobilization. At low polymer coverages, the capacity in loading is mild but in the case of high coverage the capacity is much higher. 


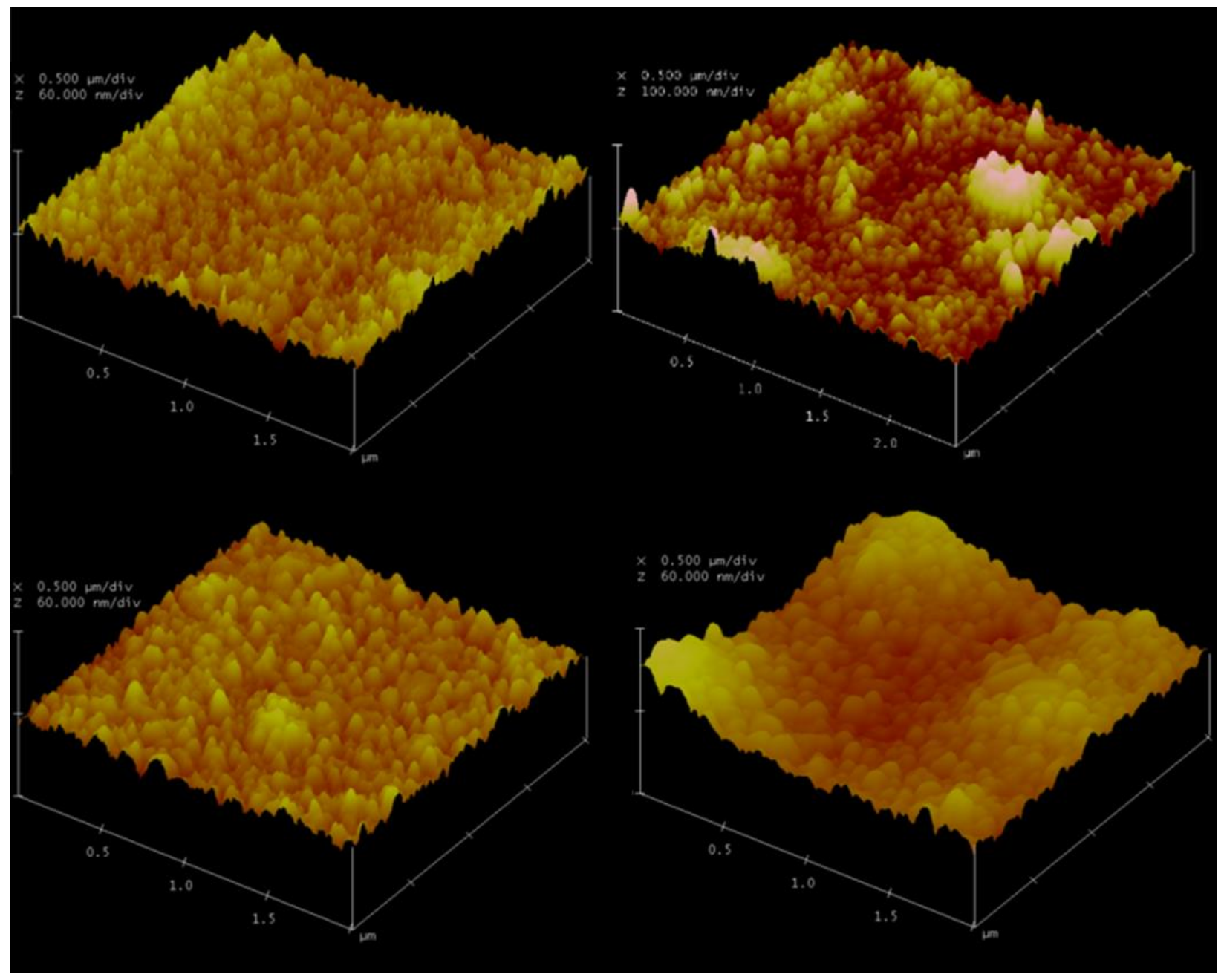

Figure 9: AFM 3-D images of the adsorbed layers. Top-left: Ag film. Top-right: Adsorbed lysozyme on Ag surface. Bottom-left: Adsorbed PtBS-b-SCPI micelles (high coverage). Bottomright: Lysozyme adsorbed on preadsorbed micelles.

AFM images from the Ag surface and the adsorbed layers are shown in figure 9 in the same lateral length-range. This 3-D representation of AFM images (figure 9) shows clearly that adsorption of PtBS-b-SCPI micelles (bottom-left) and of lysozyme (top-right) occurs on the Ag substrate. The roughness of the Ag surfaces (top-left) produces sharp edges compared to the adsorbed layers. PtBS-b-SCPI adsorption (high coverage) presents larger, smoother and more spherical formations extending from the layer compared to the Ag substrate. Since the adsorbed amounts are found to be higher than the overlapping one (table 1) we conclude that there is no 
free Ag surface i.e. the micelles have totally covered the Ag roughness. In the case of the adsorbed protein the peak morphology is between Ag and PtBS-b-SCPI in terms of sharpness but the formations are not uniformly spread on the surface[45]. This means that as long as some lysozyme has been adsorbed, the incoming lysozyme accumulates preferentially near the globules that are already on the surface. This non-uniform distribution adds long-wavelength modulation to the layer morphology. For the complexed layers (bottom-right) we observe large spherical formations and additionally long wavelength modulation of the layer. The spherical features are better-defined and bigger than the ones in pure micellar layers pointing to the possibility that lysozyme globules enter the micelles, swell them and create overlapping spherical structures.
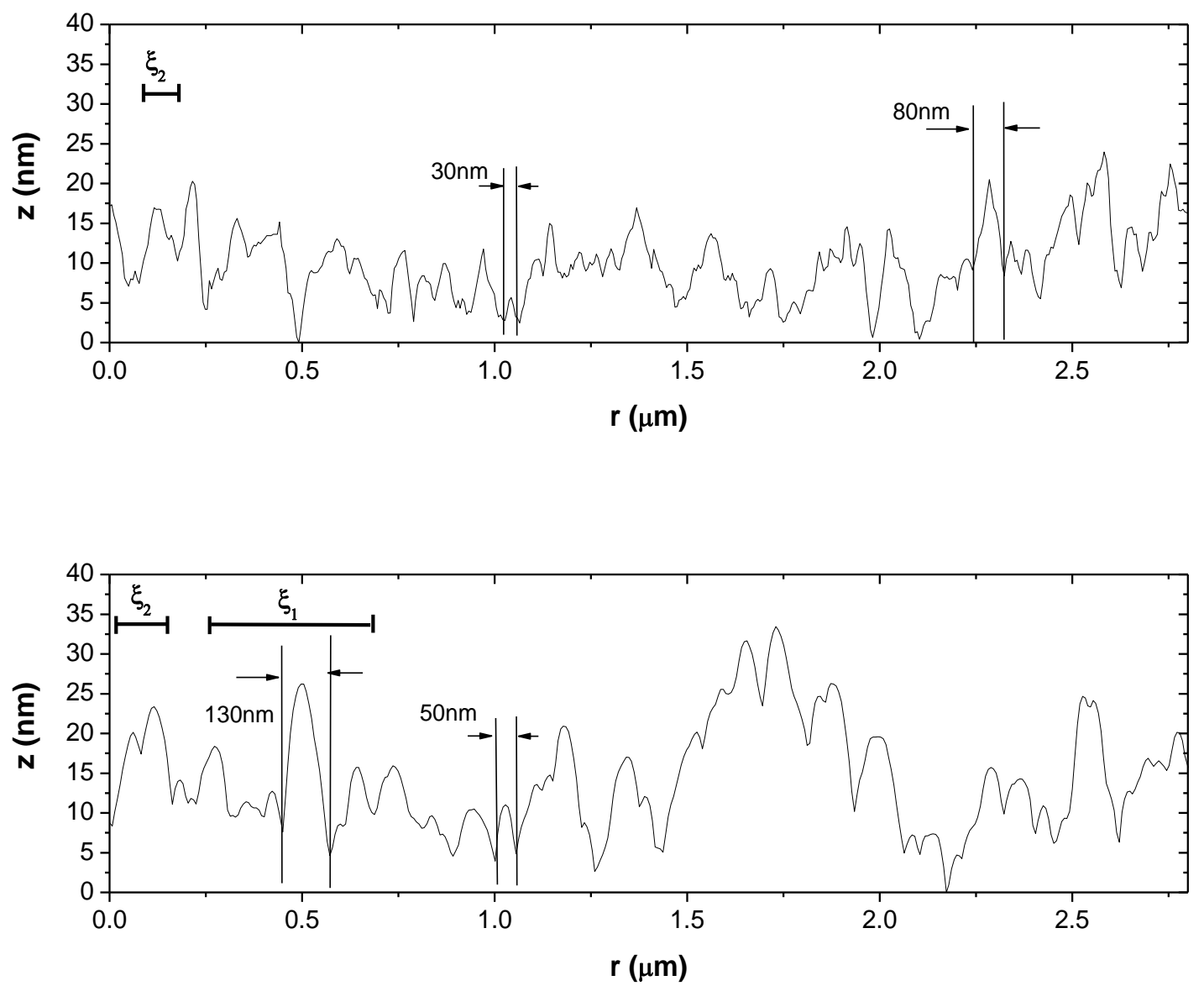

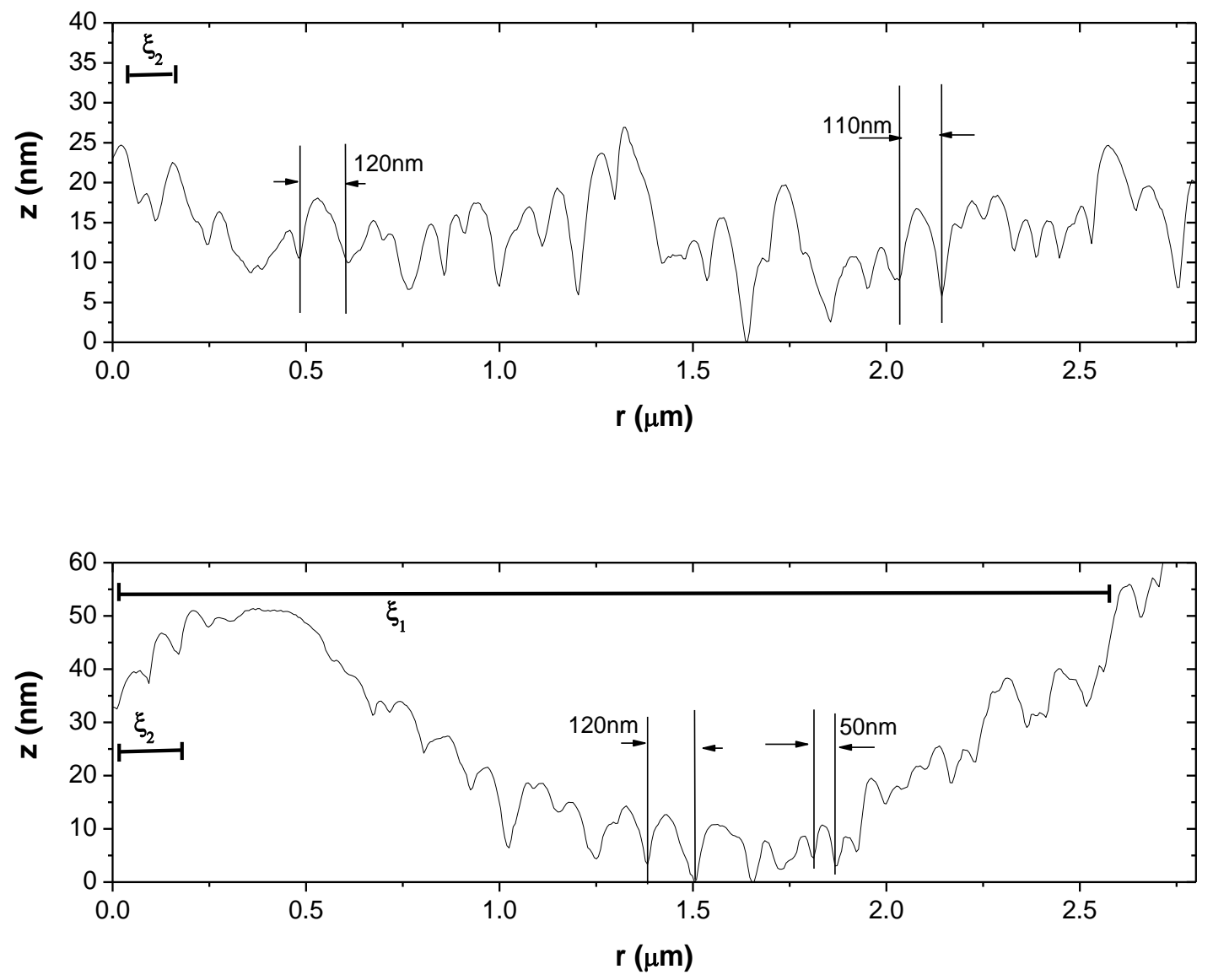

Figure 10: AFM section analysis of the adsorbed layers. Top to bottom: Ag film, adsorbed lysozyme on Ag surface, adsorbed PtBS-b-SCPI micelles (high coverage) and lysozyme adsorbed on preadsorbed micelles (mind the different $\mathrm{z}$-scale of the complexed layer). Horizontal bars present correlation lengths in absolute scale (PSD analysis discussion).

The features of the AFM images are quantitatively described by the section analysis in figure 10 . The section is taken as a diagonal of the image and $r$ is the distance along this direction. The roughness value is double in the case of adsorbed micelles $(\sigma=5.2 \mathrm{~nm})$ compared to the one of the bare Ag surface $(\sigma=2.6 \mathrm{~nm}$ ). More importantly, the rougness profile is smoother in the case of adsorbed micelles which means that the micelles have covered the Ag roughness. The in-plane 
size of a characteristic feature in the micelles layer image is $\sim 50-100 \mathrm{~nm}$. Comparing with the average intermicellar distance $\left(r_{m} \approx 40 \mathrm{~nm}\right)$ from SPR we may assume that in the adsorbed layers single micelles or overlapping micelles are observed. The height of the features (zdirecton) does not exceed $35 \mathrm{~nm}$ which means that the micelles are collapsed during the AFM experiment since their solution radius is $100 \mathrm{~nm}\left(3^{\text {rd }}\right.$ graph of figure 10). This collapsing may have been induced, at least partially, during adsorption because of contacts of the SCPI chains with the surface. We conclude that the micelles of the adsorbed PtBS-b-SCPI do not lose their integrity upon adsorption[6].

The roughness value of the adsorbed protein $(\sigma=5.2 \mathrm{~nm})\left(2^{\text {nd }}\right.$ graph of figure 10$)$ is similar to the one of the micelles. Nevertheless the height pattern is intermediate between the sharp (Ag) and the smooth (PtBS-b-SCPI) caused possibly by the size of the lysozyme globules in solution $(\sim 4.5 \mathrm{~nm})$ which is smaller than the one of the micelles. The radii of characteristic features can be up to $50 \mathrm{~nm}$ though, reflecting that proteins form aggregates on the surface. At the same time, the long-wavelength modulations are clearly depicted in the modulation of the section analysis profile.

For the complexed layer (bottom graph of figure 10) the section analysis reveals the two lengthscale profile. A short-length scale modulation which reveals spherical structures of radius ( $30-$ $60 \mathrm{~nm})$ and long-length scale modulation $(\sim 1000 \mathrm{~nm})$. These two length-scales correspond to individual micelles covered by lysozyme and islands of lysozyme respectively. The difference in height between the low and high regions can be up to $\sim 50 \mathrm{~nm}$. 


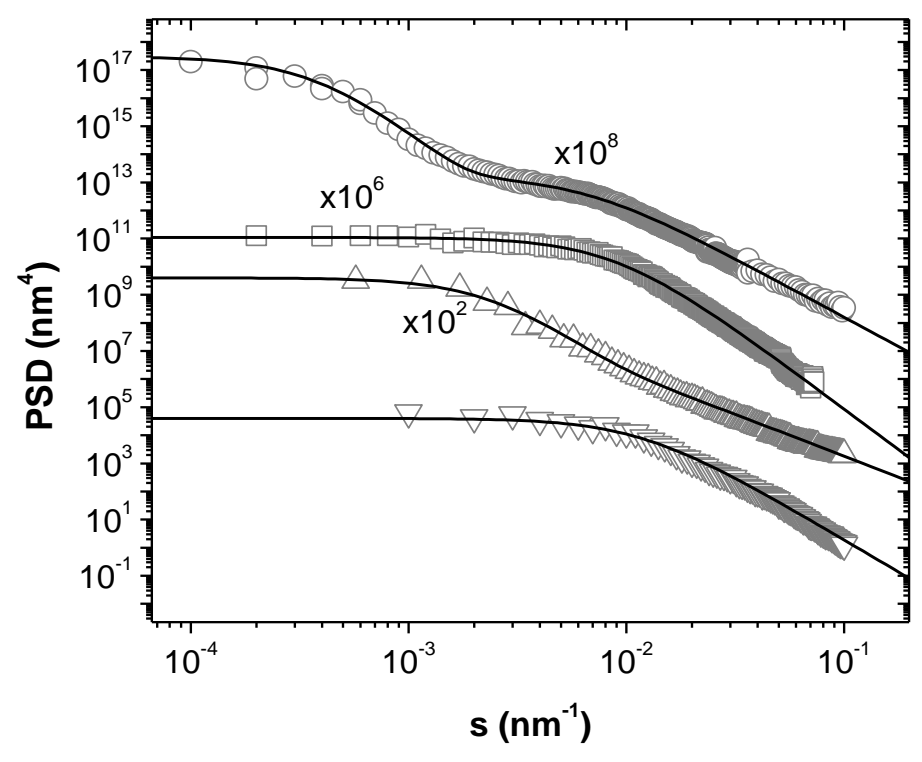

Figure 11: Radial PSD functions from the surfaces studied by AFM. From bottom to top: Ag substrate, adsorbed lysozyme, adsorbed micelles and micelles adsorbed on micellar layer. Straight lines are fittings with the model of equation 11.

Table 2: Optimized parameters for the PSD functions of the four layers studied by AFM fitted by equation 11 .

\begin{tabular}{|c|c|c|c|c|}
\hline Layer & $\xi_{\mathbf{1}}(\mathbf{n m})$ & $\mathbf{n}_{\mathbf{1}}$ & $\xi_{\mathbf{2}}(\mathbf{n m})$ & $\mathbf{n}_{\mathbf{2}}$ \\
\hline Silver & - & - & $90 \pm 10$ & $4.6 \pm 0.2$ \\
\hline Lysozyme & $410 \pm 40$ & $2.8 \pm 0.1$ & $130 \pm 10$ & $3.0 \pm 0.2$ \\
\hline Micelles & - & - & $110 \pm 10$ & $5.8 \pm 0.2$ \\
\hline Complexed & $2600 \pm 100$ & $3.1 \pm 0.1$ & $160 \pm 10$ & $4.2 \pm 0.1$ \\
\hline
\end{tabular}

The radial PSD from the four layers studied by AFM are presented in figure 11. The curves show characteristic plateaus followed by power-law behaviors [46]. For large length-scales (low svalues) PSD represents the long-range average film roughness. For small length-scales (high qvalues) PSD represents the short-range self-similar roughness [47]. The onset of the power-law 
behaviour, is a measure of the correlation length of the surface and the power-law exponent is related to the "roughness exponent" or the Hurst exponent of a self-affine surface [28]. In the case of the complexed layers and the lysozyme layer, two-length scales (long and short wavelength modulations) are found in the PSD profiles as two plateau-power/law pairs [48].

$P(s)=\frac{A}{\left(1+s^{2} \xi^{2}\right)^{n / 2}}$

Equation 11 is used for fitting the data of figure 11. The fitting parameters [48, 49] are the correlation length $(\xi)$, the roughness exponent $(n)$ and the multiplying constant $(A)$ which is related to the rms surface roughness $(\sigma)$. The results of the fits are shown in table 2. For lysozyme layers and complexed layers a two-mode model of equation 11 (linear combination) is obviously needed to fit the PSD data. The short-wavelength correlation length $\left(\xi_{2}\right)$ of lysozyme is the same (within error) with the one of the Ag layer. This shows that lysozyme globules follow the structure of the underlying surface. The fractal exponent of lysozyme layer is lower than the one of the Ag layer possibly because of the richer content in lateral features of the lysozyme layer.

The correlation length $\left(\xi_{2}\right)$ for the micellar layer is in agreement with the diameter of the largest characteristic features in the AFM images (figure 10). This correlation length increases upon addition of lysozyme which agrees with figure's 9 3-D image and highlights the fact that the individual loading with protein the spherical structures swell, overlap and create surface aggregates. The scaling exponent $\left(n_{2}\right)$ of the micellar layer is higher than the ones of $\mathrm{Ag}$ and lysozyme because the spherical features are more well-defined as shown in the 3-D AFM images. This short-wavelength scaling exponent drops upon addition of lysozyme because the protein globules introduce short-length scale complexity as in the case of Ag layer. Presence of protein globules creates islands on the $\mathrm{Ag}$ and micellar films (figures 9 and 10). This is characterized by the long-wavelength correlation length $\left(\xi_{1}\right)$ which is higher when there is underlying micellar layer compared to the bare Ag surface (table 2). 


\section{CONCLUSIONS}

We investigated the adsorption of PtBS-b-SCPI micelles on the Ag/water interface by surface plasmon resonance and atomic force microscopy. We found that the micelles irreversibly adsorb on the Ag surface and that they do not lose their integrity upon adsorption. The characteristic correlation length of the micellar layer is retained upon complexation of lysozyme with the micellar layers. The loading capacity of the micellar layers in lysozyme depends on the lysozyme solution concentration and the micellar density and structure of the underlying layer. This means that amphiphilic polyelectrolyte micelles adsorbed on solid/water interfaces can produce welldefined, tailor-made and stable substrates for engineered surface nanostructures having potential applicability in functional biointerfaces and biomaterials fabrication, enzyme immobilization, protein/DNA purification and delivery, and cell immobilization/proliferation on surfaces. The presented results also extend our understanding on synthetic/biological soft matter interactions at interfaces and produce guidelines for fine-tuning of such interactions as well as of hybrid nanostructure formation.

\section{Acknowledgement}

Authors acknowledge financial support of the work by the NANOMACRO 1129 project which is implemented in the framework of the Operational Program "Education and Life-long Learning" (Action "ARISTEIA I") and it is co-funded by the European Union (European Social Fund) and by national funds.

\section{REFERENCES}

1. Fleer, G.J., et al., Polymers at interfaces. Polymer International. 1990, London: Chapman \& Hall.

2. Stuart, M.A.C., et al., Emerging applications of stimuli-responsive polymer materials. Nat Mater, 2010. 9(2): p. 101-113.

3. Gil, E.S. and S.M. Hudson, Stimuli-reponsive polymers and their bioconjugates. Progress in Polymer Science, 2004. 29(12): p. 1173-1222.

4. Szilagyi, I., et al., Polyelectrolyte adsorption, interparticle forces, and colloidal aggregation. Soft Matter, 2014. 10(15): p. 2479-2502.

5. Hamley, I., Block Copolymers in Solution: Fundamentals and Applications. 2005, Chichester: Wiley. 
6. Mahltig, B., et al., Adsorption of block polyampholyte micelles in monolayers at the silicon water interface. Colloid and Polymer Science, 2000. 278(6): p. 502-508.

7. De Vos, W.M., J.M. Kleijn, and M.A.C. Stuart, Polymer Brushes Through Adsorption: From Early Attempts to the Ultra-Dense and Reversible "Zipper Brush", in Polymer Brushes: Substrates, Technologies, and Properties, V. Mittal, Editor. 2012, CRC Press.

8. Becker, A.L., et al., Proteins and polyelectrolytes: A charged relationship. Curr Opin Colloid Int Sci, 2012. 17: p. 90-96.

9. Jiang, B., J.B. Barnett, and B. Li, Advances in polyelectrolyte multilayer nanofilms as tunable drug delivery systems. Nanotechnol Sci Appl, 2009. 9: p. 21-27.

10. Saptarshi, S.R., A. Duschl, and A.L. Lopata, Interaction of nanoparticles with proteins: relation to bio-reactivity of the nanoparticle. Journal of Nanobiotechnology, 2013. 11(26): p. 1-12.

11. Pergushov, D.V., et al., Interpolyelectrolyte Complexes Based on Polyionic Species of Branched Topology. Adv. Polym. Sci., 2011. 241: p. 131-161.

12. Karayianni, M. and S. Pispas, Block polyelectrolyte micelles/protein mixed nanostructures in aqueous media, in Micelles: Structural Biochemistry, Formation and Functions \& Usage, D. Bradburn and T. Bittinger, Editors. 2013.

13. Bittrich, E., et al., Protein adsorption on and swelling of polyelectrolyte brushes: A simultaneous ellipsometry-quartz crystal microbalance study. Biointerphases, 2010. 5(4): p. 159-167.

14. Wijaya, E., et al., Surface plasmon resonance-based biosensors: From the development of different SPR structures to novel surface functionalization strategies. Current Opinion in Solid State and Materials Science, 2011. 15(5): p. 208-224.

15. Schwarz, S., et al., Adsorption of Polyelectrolytes with Hydrophobic Parts, in Characterization of Polymer Surfaces and Thin Films, K. Grundke, M. Stamm, and H.-J. Adler, Editors. 2006, Springer Berlin Heidelberg. p. 102-109.

16. Mrksich, M., G.B. Sigal, and G.M. Whitesides, Surface Plasmon Resonance Permits in Situ Measurement of Protein Adsorption on Self-Assembled Monolayers of Alkanethiolates on Gold. Langmuir, 1995. 11(11): p. 4383-4385.

17. Hadjichristidis, N., et al., Anionic polymerization: High vacuum techniques. Journal of Polymer Science Part A: Polymer Chemistry, 2000. 38(18): p. 3211-3234.

18. Uhrig, D. and J.W. Mays, Experimental techniques in high-vacuum anionic polymerization. Journal of Polymer Science Part A: Polymer Chemistry, 2005. 43(24): p. 6179-6222.

19. Raether, H., Surface Plasmons on Smooth and Rough Surfaces and on Gratings. 1986, Hamburg: Springer-Verlag. 
20. Koutsioubas, A.G., et al., Adsorption behavior of PS-PEO diblock copolymers on silver and alumina surfaces: A surface plasmon resonance study. Journal of Polymer Science Part B: Polymer Physics, 2006. 44(11): p. 1580-1591.

21. Ritchie, R.H., Plasma Losses by Fast Electrons in Thin Films. Phys Rev, 1957. 106: p. 874-881.

22. $\quad$ Abelès, F., Surface electromagnetic waves ellipsometry. Surface Science, 1976. 56(0): p. 237251.

23. M., B. and W. E., Principles of Optics. 1974: Cambridge University Press.

24. Ostuni, E., L. Yan, and G.M. Whitesides, The interaction of proteins and cells with selfassembled monolayers of alkanethiolates on gold and silver. Colloids and Surfaces B: Biointerfaces, 1999. 15(1): p. 3-30.

25. Green, R.J., et al., Surface plasmon resonance for real time in situ analysis of protein adsorption to polymer surfaces. Biomaterials, 1997. 18(5): p. 405-413.

26. Ordal, M.A., et al., Optical properties of the metals $\mathrm{Al}, \mathrm{Co}, \mathrm{Cu}, \mathrm{Au}, \mathrm{Fe}, \mathrm{Pb}, \mathrm{Ni}, \mathrm{Pd}, \mathrm{Pt}, \mathrm{Ag}$, Ti, and $W$ in the infrared and far infrared. Applied Optics, 1983. 22(7): p. 1099-1119.

27. Binnig, G., C.F. Quate, and C. Gerber, Atomic Force Microscope. Physical Review Letters, 1986. 56(9): p. 930-933.

28. Stone, V.W., et al., Roughness of free surfaces of bulk amorphous polymers as studied by x-ray surface scattering and atomic force microscopy. Physical Review B, 1999. 60(8): p. 5883-5894.

29. Green, R.J., et al., Surface plasmon resonance analysis of dynamic biological interactions with biomaterials. Biomaterials, 2000. 21(18): p. 1823-1835.

30. Jiang, G., et al., Signal Enhancement and Tuning of Surface Plasmon Resonance in Au Nanoparticle/Polyelectrolyte Ultrathin Films. The Journal of Physical Chemistry C, 2007. 111(50): p. 18687-18694.

31. Ekgasit, S., et al., Resonance shifts in SPR curves of nonabsorbing, weakly absorbing, and strongly absorbing dielectrics. Sensors and Actuators B: Chemical, 2005. 105(2): p. 532-541.

32. Lutkenhaus, J.L. and P.T. Hammond, Electrochemically enabled polyelectrolyte multilayer devices: from fuel cells to sensors. Soft Matter, 2007. 3(7): p. 804-816.

33. Eagen, C.F. and W.H. Weber, Modulated surface-plasmon resonance for adsorption studies. Physical Review B, 1979. 19(10): p. 5068-5082.

34. Bennett, H.E. and J.L. Stanford, Structure-related optical characteristics of thin metallic films in the visible and ultraviolet. J Res Natl Stand Sec A, 1976. 80A(4): p. 643-658.

35. Filippova, N.L., Adsorption and Desorption Kinetics of Polyelectrolytes on Planar Surfaces. Langmuir, 1998. 14(5): p. 1162-1176. 
36. Sukhishvili, S.A. and S. Granick, Kinetic regimes of polyelectrolyte exchange between the adsorbed state and free solution. The Journal of Chemical Physics, 1998. 109(16): p. 6869-6878.

37. Abraham, T., et al., Adsorption Kinetics of a Hydrophobic-Hydrophilic Diblock Polyelectrolyte at the Solid-Aqueous Solution Interface: A Slow Birth and Fast Growth Process. Macromolecules, 2000. 33(16): p. 6051-6059.

38. Raposo, M., et al., Kinetics of Adsorption of Poly(o-methoxyaniline) Self-Assembled Films. Macromolecules, 1997. 30(20): p. 6095-6101.

39. Karpovich, D.S. and G.J. Blanchard, Direct Measurement of the Adsorption Kinetics of Alkanethiolate Self-Assembled Monolayers on a Microcrystalline Gold Surface. Langmuir, 1994. 10(9): p. 3315-3322.

40. Peng, B., et al., Adsorption kinetics and stability of poly(ethylene oxide)-block-polystyrene micelles on polystyrene surface. Polymer, 2013. 54(21): p. 5779-5789.

41. Gensel, J., et al., Surface immobilized block copolymer micelles with switchable accessibility of hydrophobic pockets. Soft Matter, 2011. 7(23): p. 11144-11153.

42. Stuart, M.A.C., C.W. Hoogendam, and A.d. Keizer, Kinetics of polyelectrolyte adsorption. Journal of Physics: Condensed Matter, 1997. 9(37): p. 7767.

43. Fang, F., J. Satulovsky, and I. Szleifer, Kinetics of Protein Adsorption and Desorption on Surfaces with Grafted Polymers. Biophysical Journal, 2005. 89(3): p. 1516-1533.

44. Sukhishvili, S.A. and S. Granick, Adsorption of human serum albumin: Dependence on molecular architecture of the oppositely charged surface. The Journal of Chemical Physics, 1999. 110(20): p. 10153-10161.

45. Bhambhani, A., et al., Folding Control and Unfolding Free Energy of Yeast Iso-1-cytochrome c Bound to Layered Zirconium Phosphate Materials Monitored by Surface Plasmon Resonance. The Journal of Physical Chemistry B, 2008. 112(30): p. 9201-9208.

46. Rönnow, D., J. Isidorsson, and G.A. Niklasson, Surface roughness of sputtered ZrO films studied by atomic force microscopy and spectroscopic light scattering. Physical Review E, 1996. 54(4): p. 4021-4026.

47. Gredig, T., E.A. Silverstein, and M.P. Byrne, Height-Height Correlation Function to Determine Grain Size in Iron Phthalocyanine Thin Films. Journal of Physics: Conference Series, 2013. 417(1): p. 012069.

48. Senthilkumar, M., et al., Characterization of microroughness parameters in gadolinium oxide thin films: A study based on extended power spectral density analyses. Applied Surface Science, 2005. 252(5): p. 1608-1619. 
49. Palasantzas, G., Roughness spectrum and surface width of self-affine fractal surfaces via the Kcorrelation model. Physical Review B, 1993. 48(19): p. 14472-14478. 